Robert N. Sladen MBCHB, MRCP(UK), FRCPC(C)

\title{
Pharmacology of shock: an update
}

\section{Pathogenesis of shock}

Appropriate pharmacotherapy in shock is facilitated by a concise hemodynamic analysis of its pathogenesis. ${ }^{1} \mathrm{~A}$ first step is to consider the systemic circulation as a simple circuit. A pump, the heart, is subtended by an arterial system that governs systemic vascular resistance (SVR) and afterload, a capillary bed that provides tissue nutrition and metabolite removal, and a venous system that determines venous return and preload. Shock may be defined simply as inadequate perfusion of vital capillary beds. It may be further classified by its primary clinical manifestation as vasoconstricted or vasodilated, and analyzed hemodynamically by "Ohm's Law of the Circulation", i.e., blood pressure (BP) is the product of cardiac output $(\mathrm{CO})$ and SVR:

$\mathrm{BP}=\mathrm{CO} \times \mathrm{SVR}$.

Vasoconstricted shock may be caused by cardiogenic shock or hypovolemic shock. The primary event is either pump failure or hypovolemia leading to a fall in $\mathrm{CO}$ and hypotension ("low output shock"). As a consequence of baroreceptor-induced sympathetic stimulation, there is both arterial and venous constriction, which seeks to restore BP to normal levels. Because the cerebral and coronary circulations are relatively devoid of alpha-adrenergic receptors, blood flow to the heart and brain is conserved at the expense of other vital organs, notably the kidney, gut and lungs. At the arteriolar and venular level, there is constriction of the pre-and postcapillary sphincters which further impairs tissue perfusion.

Vasodilated shock is most often associated with sepsis or the systemic inflammatory response syndrome (SIRS). The primary event is profound cutaneous vasodilation and decrease in SVR, leading to a fall in BP. As a consequence of baroreceptor-induced sympathetic stimulation, $\mathrm{CO}$ increases in an attempt to restore BP to normal levels ("high output shock"). In fact both the terms vasodilated and high output are misnomers. Although SVR is decreased, many vascular beds (renal, splanchnic and pulmonary) are severely vasoconstricted, and circulating depressant factors cause substantial myocardial dysfunction. A more accurate and descriptive term is "distributive shock".

Protracted shock of any etiology culminates in the accumulation of lactic acid. This has important consequences for the vascular bed, as will be detailed later. Lactic acid dilates the more sensitive pre-capillary sphincter, which allows blood to reflow into the capillary bed but not out of it. Hydrostatic pressure builds up and increases capillary leak and interstitial edema, further impairing cellular nutrition. The blood that remains in the capillaries becomes increasingly viscous and sludges, triggering platelet activation and disseminated intravascular coagulation (DIC). Lactic acidosis and DIC are hallmarks of all forms of protracted shock.

\section{Pharmacotherapy of cardiogenic shock}

The development of cardiogenic shock implies the loss of more than $40 \%$ of functioning myocardium. It may develop as a consequence of acute myocardial infarction, acute intraoperative ischemia and reperfusion injury (e.g., during cardiopulmonary bypass). Stunned myocardium refers to acute loss of function due to ischemia that may recover with time. Hibernating myocardium refers to chronic loss of function due to ischemia (usually identified by positron emission tomography scan) that may be recruitable with revascularization. ${ }^{2}$ End-stage heart disease due to ischemic, viral or idiopathic cardiomyopathy ultimately culminates in a state of chronic cardiogenic shock with progressive multisystem organ dysfunction.

Ischemic cardiogenic shock that develops after cardiopulmonary bypass is a classic example of a vicious cycle. Decreased perfusion leads to cardiac injury, resulting in decreased stroke volume and hypotension. Catecholamine release (or administration) induces tachycardia that further impairs myocardial oxygen balance and exacerbates acute myocardial injury. ${ }^{1}$

In chronic cardiomyopathy with sustained release (or administration) of catecholamines there is progressive beta receptor downregulation. ${ }^{3}$ In the normal myocardium, about $80 \%$ of the inotropic response is mediated by beta- 1 receptors and $20 \%$ by beta- 2

\footnotetext{
From the Department of Anesthesiology, College of Physicians and Surgeons of Columbia University, New York, New York, USA. Address correspondence to: Dr. Robert N. Sladen, Department of Anesthesiology, PH 527B, College of Physicians and Surgeons of Columbia University, 630 W. 168th Street, New York, NY 10032, USA. Phone: 212-305-8633; Fax: 212-305-8287;

E-mail: rs543@columbia.edu
} 
receptors. In chronic cardiomyopathy, the proportion mediated by beta- 2 receptors increases to $40 \%$, and the response to beta-l adrenergic agents such as dopamine becomes progressively impaired, while that to beta-2 agents such as dobutamine becomes progressively more important. ${ }^{4}$

Hemodynamic management of impaired CO should be organized in an orderly sequence, with reassessment to response at each step. Establishment of normal rate and rhythm is an essential first step in creating a stable milieu in which the subsequent interventions may be applied. Inadequate preload should be augmented, excessive preload decreased. Inotropic support is then applied, with reduction of increased SVR, or augmentation of inadequate SVR (see below). ${ }^{5}$

There are two groups of inotropic agents: beta adrenergic agonists and phosphodiesterase (PDE) III inhibitors.

\section{Beta adrenergic agonists}

Beta-adrenergic agents are administered to enhance inotropy (force of contraction), but will also enhance chronotropy (heart rate), dromotropy (conduction velocity) and bathmotropy (ectopic beats and rhythms). The dromotropic effect is illustrated by the increase in atrio-ventricular conduction and heart rate when catecholamines are administered to patients with atrial fibrillation. It should be expected that any beta-adrenergic agent may induce tachycardia and tachyarrhythmias.

It is useful to classify beta-adrenergic agents according to their effect on the peripheral circulation. Inoconstrictors confer inotropy with alpha-adrenergic induced vasoconstriction, and inodilators confer inotropy with beta- 2 adrenergic induced vasodilation.

Inoconstrictors include the naturally occurring catecholamines, norepinephrine, epinephrine and dopamine. Norepinephrine is a potent, direct-acting beta- 1 adrenergic agent as well as alpha adrenergic vasoconstrictor. This makes it especially useful in states of vasodilated shock, where myocardial depression and profound vasodilation coexist. Because of its beta-2 adrenergic action, epinephrine has a more unpredictable effect on SVR and is more prone to precipitate tachycardia and tachyarrhythmias.

The beta adrenergic inodilators are all synthetic derivatives of dopamine, and include dobutamine, dopexamine and isoproterenol. Dobutamine is a potent direct acting beta adrenergic agent, whose beta- 2 mediated inotropic effect is particularly helpful in chronic cardiomyopathy. ${ }^{6}$ Its beta- 2 vasodilator effects also provide pulmonary artery vasodilation that may be helpful in weaning patients off cardiopul- monary bypass with acute right ventricular failure. Dopexamine is an investigational agent (available in Europe) which acts predominantly through beta-2 mediated afterload reduction, with secondary release of norepinephrine that enhances its inotropic effect. ${ }^{7}$ Isoproterenol is limited by its potent chronotropic effect, which precedes its inotropic effect, and its use is largely confined to pharmacologic pacing.

\section{PDE inbibitors}

Administration of PDE inhibitors has particular advantages in patients with cardiomyopathy or protracted cardiac failure, who have down-regulation of beta receptors. ${ }^{8}$ Their action in preventing the breakdown of cyclic adenosine monophosphate (cAMP) is independent of the beta receptor. ${ }^{9}$ In cardiac muscle the increase in cAMP promotes intracellular calcium release and muscle contraction (inotropic effect). In smooth muscle it has the opposite effect, preventing the release of calcium and promoting smooth muscle relaxation (vasodilation). The PDE inhibitors function as true inodilators, and provide afterload reduction for both the left and right ventricles. They also promote diastolic relaxation (positive lusitropic effect), which enhances myocardial oxygen balance. They are less bathmotropic (arrhythmogenic) than catecholamines. Finally, and very importantly, their combination with catecholamines provides a synergistic increase in stroke volume, i.e., cAMP is increased by a combination of increased production (catecholamines) and decreased breakdown (PDE inhibition). ${ }^{10}$

Milrinone has largely replaced amrinone as the archetype PDE inhibitor. It is about 15 times more potent than amrinone, there is a lower risk of thrombocytopenia and it has a shorter elimination half-life (2.4 hr vs $5.8 \mathrm{hr}$ ). Unlike amrinone, milrinone does not decompose in dextrose solutions and does not need protection from the light, and it contains no bisulfite, which can induce asthma in susceptible subjects. The loading dose, which should be administered over at least ten minutes, is $50 \mu \mathrm{g} \cdot \mathrm{kg}^{-1}$, and the maintenance infusion ranges from $0.375-0.75 \mu \mathrm{g} \cdot \mathrm{kg}^{-1} \cdot \mathrm{min}^{-1}$.

The hemodynamic effects of milrinone are mediated by a combination of afterload reduction and enhanced inotropy, and result in about 30\% increase in CO. ${ }^{11}$ Compared to dobutamine, its effects are greater on the SVR but there is less tachycardia and fewer tachyarrhythmias.

There are certain limitations and caveats with the use of PDE inhibitors. Careful loading is required because acute hypotension can occur, especially in hypovolemic patients. Fluid challenges should be administered at the first signs of hypotension, and the loading infusion 
should be given over a longer period of time (e.g., 20-30 min) in unstable patients. Because of the large volume of distribution, the loading dose should be increased when it is given during cardiopulmonary bypass. It has been demonstrated that institution of the maintenance dose alone, without a loading dose, achieves a clinical effect within $30 \mathrm{~min} .{ }^{12}$

Although milrinone is rapidly redistributed, unlike catecholamines it is eliminated much more slowly and depends on the kidneys for about $40 \%$ of its excretion. It accumulates with time and has a long duration of action, especially in patients with renal dysfunction, in whom the maintenance dose should be decreased. ${ }^{13}$ Finally, these drugs are not cheap. In the US, a 48-hr course of milrinone has an acquisition cost of about $\$ 500$.

\section{Counterpulsation}

Intra-aortic balloon counterpulsation provides an important means of restoring myocardial oxygen balance. During early to mid-diastole, balloon inflation augments coronary perfusion pressure; at end-diastole balloon deflation decreases myocardial afterload. Decreased systolic pressure is required for cardiac ejection during the following beat. No combination of inotropic, vasodilator or vasoconstrictor therapy can truly mimic the advantage provided by intra-aortic counterpulsation. ${ }^{14}$ Indeed, the error that is occasionally made after cardiopulmonary bypass is to delay the insertion of an intra-aortic balloon. Excessive persistence with pharmacotherapeutic support alone may expose the myocardium to a period of hypoperfusion, tachycardia and increased contractility that exacerbates myocardial ischemia and creates a vicious cycle.

\section{Pharmacotherapy of vasodilated shock}

Vasodilated shock describes a clinical entity characterized by severe hypotension caused by refractory vasodilation. ${ }^{15}$ However, vasodilation is not uniform, and there is associated hypoperfusion of the renal, splanchnic and pulmonary vascular beds (distributive shock). In the early phase, cardiac index may be increased above normal, but ultimately myocardial depression becomes more and more apparent. The archetype of vasodilated shock is septic shock, in which a profound decrease in SVR, mediated by endotoxin and cytokines, is the presenting feature. However, vasodilated shock may occur in many other instances, including protracted cardiogenic shock, protracted hypovolemic shock, massive contact activation (induced by cardiopulmonary bypass or ventricular assist device) or as a consequence of milrinone-induced vasodilation. ${ }^{16}$

There are now considered to be at least three important mechanisms of vasodilatory shock: 1) opening of potassium-adenosine triphosphate $\left(\mathrm{K}_{\mathrm{ATP}}\right)$ channels; 2$)$ acute arginine vasopressin (AVP) deficiency; and 3) widespread activation of inducible nitric oxide synthase (iNOS) and massive release of nitric oxide $(\mathrm{NO}) .{ }^{15}$

\section{Opening of $K_{A T P}$ channels}

Vasoconstrictors such as norepinephrine and angiotensin stimulate G-protein coupled endothelial receptors that open cell membrane calcium channels and promote an influx of calcium. This, together with the release of calcium from intracellular stores, activates a kinase that enhances the phosphorylation of myosin and results in muscle contraction and vasoconstriction. In the presence of intracellular acidosis, lactate accumulation and ATP depletion, membrane $\mathrm{K}_{\mathrm{ATP}}$ channels open and allow an efflux of potassium. This hyperpolarizes the membrane and closes the calcium channels, resulting in vasodilation that is refractory to norepinephrine..$^{15}$

\section{Acute AVP deficiency}

A few years ago it was serendipitously found that a relatively modest dose of AVP by infusion (0.5-6 units.hr- $\left.{ }^{-1}\right)$ results in remarkable improvement in BP and urine flow in patients with vasodilated septic shock, allowing rapid tapering of catecholamines. ${ }^{17}$ Normally, AVP exerts an antidiuretic effect on $\mathrm{V}_{2}$-receptors in the distal renal tubule and collecting duct in response to tiny (1\%) elevations in serum osmolality. Plasma AVP over the range of 1 through $5 \mathrm{pg} \cdot \mathrm{L}^{-1}$ increases urine osmolality from 300 to $1200 \mathrm{mOsm} \cdot \mathrm{kg}^{-1}$. Severe hypotension induces a baroreceptor response that releases large amounts of AVP from the posterior pituitary. Plasma AVP of 10 through $200 \mathrm{pg} \cdot \mathrm{L}^{-1}$ exerts a vasoconstrictor effect on $\mathrm{V}_{1}$ receptors in vasculature and helps to restore $\mathrm{BP}$. However, it was found that patients in severe vasodilated septic shock had paradoxically low plasma AVP (about 3 $\left.\mathrm{pg} \cdot \mathrm{L}^{-1}\right) .{ }^{17}$ Subsequently it has been demonstrated in animal models of hemorrhagic shock that within an hour of the induction of profound hypotension, AVP stores in the posterior pituitary become quite depleted. ${ }^{15}$ Thus, the response to infused AVP is in part accounted for by the restoration of plasma AVP to appropriate levels for the degree of hypotension.

In addition, AVP appears to block the $\mathrm{K}_{\mathrm{ATP}}$ channel, thereby restoring membrane polarity and vascular responsiveness to catecholamines and angiotensin. AVP also has a salutary effect on renal function because it preferentially constricts the efferent arteriole, thereby enhancing glomerular filtration pressure, filtration rate and urinary flow.

There are certain important caveats regarding infusion of AVP. It is contraindicated in cardiogenic shock and should not be used to "make BP" in situations of 
low $\mathrm{CO}$ or hypovolemia. Excessive dosing may lead to unwanted acral vasoconstriction, "blue fingers and toes" and even necrosis. High doses may also cause unwanted coronary and mesenteric vasoconstriction. Apparent "vasopressin dependency" may result if an attempt is made to wean AVP too rapidly.

\section{Massive release of $\mathrm{NO}$}

Endogenous NO is formed by the action of nitric oxide synthase (NOS) on the amino acid arginine. ${ }^{18} \mathrm{NO}$ in turn activates soluble guanosine cyclase (sGC), which acts on guanosine triphosphate to produce cyclic guanosine monophosphate, which induces vasodilation. A form of constitutive NOS, endothelial NOS (eNOS) is responsible for the continuous ("tonic") production of low levels of NO that maintain vascular patency. iNOS is activated by the action of cytokines on macrophages in sepsis or the SIRS and produces huge amounts of NO over protracted periods of time. This induces profound systemic vasodilation refractory to norepinephrine, and also inhibits beta-adrenergic inotropy and results in myocardial depression.

In animal models of sepsis, hypotension is reversed and response to catecholamines restored by NOS inhibitors. ${ }^{19,20}$ However, mortality is increase because non-selective NOS inhibitors (e.g., L-NAME, LNMMA) also suppress eNOS, thus, impairing tonic vasodilation and tissue oxygen delivery. Studies using selective iNOS inhibitors show promise in decreasing the inflammatory and vasodilator response to sepsis while maintaining tissue oxygen delivery. ${ }^{21}$

\section{References}

1 Stafford Smith M, Kaemmer DD, Sladen RN. Low cardiac output states: drugs, intra-aortic balloon and ventricular assist devices. In: Cardiac, Vascular and Thoracic Anesthesia. Youngberg JA, Lake CL, Roizen MF, Wilson RS (Eds.). New York: Churchill Livingstone, 2000; 436-65.

2 Cooper HA, Braunwald E. Clinical importance of stunned and hibernating myocardium. Coron Artery Dis 2001; 12: 387-92.

3 Bristow MR, Ginsburg R, Minobe W. Decreased catecholamine sensitivity and B-adrenergic-receptor density in failing human hearts. New Engl J Med 1982; 307: 205-11.

4 Brodde OE, Schuler S, Kretsch R, et al. Regional distribution of b-adrenoceptors in the human heart: coexistence of function of bl- and b2-adrenoceptors in both atria and ventricles in severe congestive cardiomyopathy. J Cardiovasc Pharmacol 1986; 8: 1235-42.

5 Tempe DK, Virmani S. Pharmacologic support of circulation in patients undergoing cardiac surgery. J
Indian Med Assoc 1999; 97: 411-8.

6 Majerus TC, Dasta JF, Bauman JL, et al. Dobutamine; ten years later. Pharmacotherapy 1989; 9: 245-59.

7 Fitton A, Benfield P. Dopexamine hydrochloride. A review of its pharmacodynamic and pharmacokinetic properties and therapeutic potential in acute cardiac insufficiency. Drugs 1990; 39: 308-30.

8 Siostrzonek P, Koreny M, Delle-Karth G, et al. Milrinone therapy in catecholamine-dependent critically ill patients with heart failure. Acta Anaesthesiol Scand 2000; 44: 403-9.

9 Alousi AA, Johnson DC. Pharmacology of the bipyridines: amrinone and milrinone. Circulation 1986; 73: III10-24.

10 Royster RL, Butterworth JFT, Prielipp RC, et al. Combined inotropic effects of amrinone and epinephrine after cardiopulmonary bypass in humans. Anesth Analg 1993; 77: 662-72.

11 Shipley JB, Tolman D, Hastillo A, et al. Milrinone: basic and clinical pharmacology and acute and chronic management. Am J Med Sci 1996; 311: 286-91.

12 Baruch L, Patacsil P, Hameed A, et al. Pharmacodynamic effects of milrinone with and without a bolus loading infusion. Am Heart J 2001; 141: 266-73.

13 Taniguchi T, Shibata K, Saito S, et al. Pharmacokinetics of milrinone in patients with congestive heart failure during continuous venovenous hemofiltration. Intensive Care Med 2000; 26: 1089-93.

14 Kontoyannis DA, Nanas JN, Toumanidis ST, et al. Severe cardiogenic shock, after cardioversion, reversed by the intraaortic balloon pump. Intensive Care Med 2000; 26: 649.

15 Landry DW, Oliver JA. The pathogenesis of vasodilatory shock. N Engl J Med 2001; 345: 588-95.

16 Gold JA, Cullinane S, Chen J, et al. Vasopressin as an alternative to norepinephrine in the treatment of milrinone-induced hypotension. Crit Care Med 2000; 28: 249-52.

17 Landry DW, Levin HR, Gallant EM, et al. Vasopressin pressor hypersensitivity in vasodilatory septic shock. Crit Care Med 1997; 25: 1279-82.

18 Steudel W, Hurford WE, Zapol WM. Inhaled nitric oxide: basic biology and clinical applications. Anesthesiology 1999; 91: 1090-121.

19 Stewart TE, Zhang H. Nitric oxide in sepsis. Respir Care 1999; 44: 308-14.

20 Landin L, Lorente JA, Renes E, et al. Inhibition of nitric oxide synthesis improves the vasoconstrictive effect of noradrenaline in sepsis. Chest 1994; 106: 250-6.

21 Soejima K, Traber LD, Schmalstieg FC, et al. Role of nitric oxide in vascular permeability after combined burns and smoke inhalation injury. Am J Respir Crit Care Med 2001; 163: 745-52. 